\title{
Getting drugs into Gram-negative bacteria: Rational rules for permeation through general porins
}

Silvia Acosta-Gutiérrez ${ }^{1}$, Luana Ferrara ${ }^{2}$, Monisha Pathania ${ }^{3}$, Muriel Masi $^{4}$, Jiajun Wang $^{5}$, Igor Bodrenko ${ }^{1}$, Michael Zahn ${ }^{3}$, Mathias Winterhalter ${ }^{5}$, Robert A. Stavenger ${ }^{6}$, Jean-Marie Pagès ${ }^{4}$, James H. Naismith ${ }^{7}$, Bert van den Berg ${ }^{3}$, Malcolm G. P. Page ${ }^{5}$ \& Matteo Ceccarelli ${ }^{* *}$

\author{
Affiliations \\ ${ }^{1}$ Department of Physics, University of Cagliari, Cittadella Universitaria di \\ Monserrato, SP Monserrato-Sestu Km 0.8, 09042 Monserrato (CA). \\ ${ }^{2}$ Biomedical Sciences Research Complex, University of St Andrews, St Andrews, \\ KY16 9RH, United Kingdom \\ ${ }^{3}$ Institute for Cell and Molecular Biosciences, The Medical School, Newcastle \\ University, Newcastle upon Tyne NE2 4HH, United Kingdom. \\ 4 UMR_MD1 Inserm U1261, Membranes et Cibles Thérapeutiques, 27 Bd Jean \\ Moulin, Aix-Marseille Université, Facultés de Pharmacie et de Médecine, 13005 \\ Marseille, France \\ ${ }^{5}$ Department of Life Sciences and Chemistry, Jacobs University Bremen, 28719 \\ Bremen, Germany. \\ ${ }^{6}$ Antibacterial DPU, GlaxoSmithKline, 1250 Collegeville Rd., Collegeville, \\ Pennsylvania, PA 19426, USA \\ ${ }^{7}$ Division of Structural Biology, Nuffield Department of Medicine, Roosevelt Drive, \\ Oxford, OX3 7BN, United Kingdom \\ Research Complex at Harwell, Rutherford Laboratory, Didcot, OX11 0FA, United \\ Kingdom \\ Corresponding author: Matteo Ceccarelli, matteo.ceccarelli@dsf.unica.it
}

Small, hydrophilic molecules, including most important antibiotics in clinical use, cross the Gram-negative outer membrane through the water-filled channels provided by porins. We have determined the X-ray crystal structures of the principal general porins from three species of Enterobacteriaceae, namely Enterobacter aerogenes, Enterobacter cloacae and Klebsiella pneumoniae and determined their antibiotic permeabilities as well as those of the orthologues from Escherichia coli. Starting from the structure of the porins and molecules we propose a physical mechanism underlying transport and condense it in a computationally efficient scoring function. The scoring function shows good agreement with in-vitro penetration data and will enable the screening of virtual databases to identify molecules with optimal permeability through porins and help to guide the optimization of antibiotics with poor permeation.

Keywords: bacteria, antibiotics, rational design, porins structure, transport, scoring function. 
Drug-resistant bacterial infections are a significant public health issue globally and without concrete actions and innovation there is a threat of returning to 'preantibiotic' era ${ }^{1-3}$. Gram-negative bacteria in particular have significant intrinsic resistance to many classes of antibiotics owing to their outer membrane (OM) which imposes a physical barrier that limits the range of molecules that are taken up into the bacteria ${ }^{4-6}$. This has been recognized as a contributing factor to the difficulty in finding potent new antibacterial agents acting against these organisms ${ }^{7-9}$. Analyses of compounds with antibacterial activity has shown that marketed antibiotics, and analogues, that act against Gram-negative bacteria have restricted ranges of physicochemical properties (e.g. size and lipophilicity), although wider ranges of properties have been observed for compounds that act via non-specific mechanisms, e.g. membrane disruption ${ }^{10-12}$. The emphasis on molecular properties alone was not sufficient to provide clear determinant rules for transport. In part, this is because such approach does not consider the individual mechanisms of translocation across the outer membrane and thus blurs the details of distinctly different transport routes. Many antibiotics acting against Gram-negative bacteria are polar molecules and most exploit the water-filled channels provided by outer-membrane porins (OMP) to enter the cell ${ }^{5,6}$. The transport of $\beta$-lactams through the general porins of Escherichia coli has long been used as a model system to investigate the outer-membrane translocation of hydrophilic molecules. Transport has been studied by measuring influx rates into intact cells ${ }^{13-15}$ and by monitoring liposome swelling using reconstituted, purified proteins ${ }^{16}$. The interactions between porins and antibiotics have been studied using electrophysiology and all-atom molecular dynamics simulations to provide a molecular description of the translocation events ${ }^{17-19}$. Recent efforts have been made to construct a quantitative model of drug accumulation into E. coli cells considering both influx and efflux ${ }^{14,20,21}$. In this picture, porins function as non-specific channels for the passive diffusion of drugs across the OM and steady-state intracellular drug concentrations are balanced by the specificity and efficiency of transmembrane efflux pumps $^{20,22-24}$.

The porins of Enterobacteriaceae share high levels of sequence identity with the archetypal OmpF porin of E.coli. The channel has an hourglass shape ${ }^{25,26}$, with a narrow constriction region (CR) and segregation of charged residues on opposite sides of the pore that creates a strong transverse electric field across the $\mathrm{CR}{ }^{27-29}$. Although there is high overall sequence similarity, small changes in the CR are known to perturb small molecule permeation ${ }^{30}$ and therefore specific knowledge of structural details of the individual channels, and how these influence transport, is essential.

Here we determined the crystal structures of OmpF and OmpC orthologues from three clinically relevant ${ }^{31-33}$ Gram-negative species, namely E. cloacae, E. aerogenes and $K$. pneumonia, and combined these with the known E. coli structures to provide a substantial basis for defining channel properties. We have used a set of $\beta$-lactam antibiotics, representing the most used classes, including analogues with a range of shapes and sizes, to provide a test set for investigating pore permeability. We studied the interaction and transport of this test set with the eight structurally characterized enterobacterial porins. Combining this data set with molecular dynamics simulations, we have defined the physical mechanism underlying transport and condense it into a scoring function, which accurately predicts permeability through porins. Moreover, we have shown that applying this scoring function to a small set of molecules unrelated to the training set provides results that are consistent with the published whole cell bacterial accumulation data ${ }^{10}$. 


\section{RESULTS}

\section{The porin architecture}

We determined the crystal structures of OmpF and OmpC orthologues from Enterobacter aerogenes (Omp35 and Omp36), Enterobacter cloacae (OmpE35) and Klebsiella pneumoniae (OmpK35 and OmpK36). The structure of E. cloacae OmpE36 has been solved recently ${ }^{34}$, and the structures of the E. coli orthologues are also known ${ }^{25,26}$. The porins share on average $~ 70 \%$ sequence identity (Supplementary Fig. 1, Supplementary Table 1). As expected, the overall structures (Supplementary Fig. 2) are very similar and consist of trimers of sixteen-stranded $\beta$-barrels, each with a CR halfway down the central axis formed by the extracellular loop L3 (Fig. 1, Supplementary Fig. 2). The segregation of charged residues on opposite sides of the internal walls creates a strong transverse electric field that plays an important role in stabilizing the desolvated form of polar molecules, thereby aiding their translocation ${ }^{27-29}$. The acidic residues from the CR of OmpF (Fig. 1b), D113, E117, D121 from L3, are conserved in all structures (Supplementary Fig. 1), while some differences were found for the residues forming the basic ladder on the opposing face, presumably modulating the transverse field in the different orthologues (Supplementary Fig. 1).

a

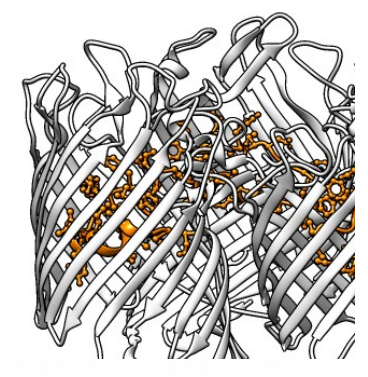

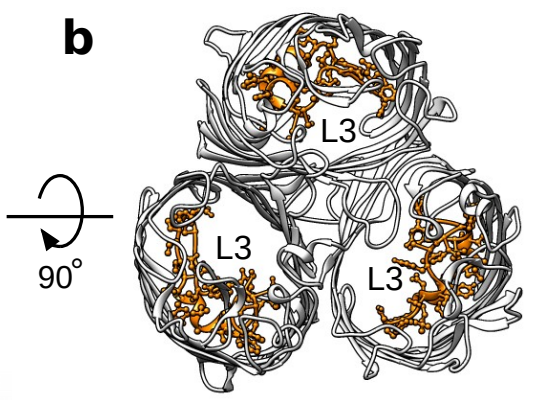
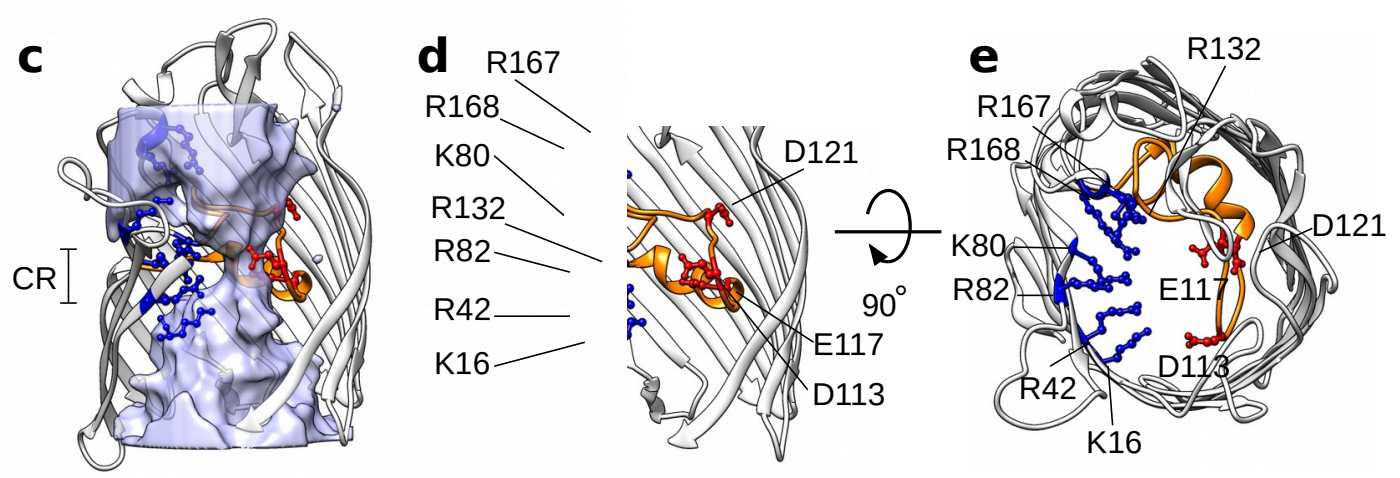

Figure 1 | The Major Outer Membrane Porin F/C architecture. The main features of the OmpF/OmpC orthologues are depicted for OmpF (E.coli): a, trimeric arrangement depicted as ribbon (Chimera software ${ }^{35}$ ), $\mathbf{b}$, top view of the trimer with the loop L3 highlighted in orange balls and sticks, c, for one of the monomers the empty lumen (calculated from all-atom simulations) is highlighted as a purple surface and the CR is indicated. $\mathbf{d}$, The main charged residues are depicted as balls and sticks and coloured according to their charge, e, top-view of $\mathbf{d}$.

\section{Structural determinants for permeability}

We modelled each porin in trimeric state embedded in a pre-equilibrated phospholipid bilayer (POPC) with a $200 \mathrm{mM} \mathrm{KCl}$ bath solution and performed all-atom MD simulations. We calculated the average geometrical cross section (Fig. 1c, 
Supplementary Fig. 3a-c) and the average internal electrostatic field ${ }^{28}$ (Supplementary Fig. 3d-f) and potential (Supplementary Fig. 3g-i) from 600ns-long trajectories. As expected, the pore radii decrease dramatically in the CR (Supplementary Fig. 3a-c). The intensity of the internal electric field is strongest at the CR and perpendicular to the axis of diffusion (transversal component) (Supplementary Fig. 3d-f). In contrast, the longitudinal component is negligible in the CR for all structures. Despite the structural similarities, we found substantial differences between the calculated electric fields. OmpC and orthologues Omp36, OmpE36, OmpK36 possess a smaller pore radius, lower conductance, and a lower intensity of the transversal electric field compared to OmpF and orthologues Omp35, OmpE35, OmpK35 (Table 1). Further, the OmpC-like proteins are more cationselective than OmpF-like (Table 1).

\begin{tabular}{|c|c|c|c|c|c|}
\hline Porin & $\begin{array}{l}\text { Conductivit } \\
\text { y } \\
1 \mathrm{MKCl} \\
+50 \mathrm{mV} \\
\text { (nS) } \\
\end{array}$ & $\begin{array}{c}\text { Selectivity } \\
\mathbf{P}_{\mathrm{K}}^{+} / \mathbf{P}_{\mathrm{Cl}^{-}} \mathbf{0 . 1} \\
\text { vs. } 1 \mathrm{M} \text { KCl }\end{array}$ & $\begin{array}{c}\text { Minimum } \\
\text { radius } \\
(\AA)\end{array}$ & $\begin{array}{c}\text { Transversa } \\
\text { l } \\
\text { electric } \\
\text { field } \\
\text { ( mV/ } \mathrm{A}) \\
\end{array}$ & $\begin{array}{c}\text { Electrostatic } \\
\text { potential } \\
\text { ( kcal/mol) }\end{array}$ \\
\hline OmpF & $4.0 \pm 0.2$ & 1.33 & $3.1 \pm 1.1$ & $25.2 \pm 0.56$ & $-1.3 \pm 0.06$ \\
\hline OmpC & $2.9 \pm 0.1$ & 2.20 & $2.8 \pm 1.1$ & $15.6 \pm 0.39$ & $-0.5 \pm 0.03$ \\
\hline Omp35 & $3.9 \pm 0.5$ & 1.72 & $3.6 \pm 1.7$ & $22.8 \pm 0.78$ & $-0.5 \pm 0.11$ \\
\hline Omp36 & $3.0 \pm 0.2$ & 2.22 & $3.0 \pm 1.3$ & $14.9 \pm 0.01$ & $-0.7 \pm 0.03$ \\
\hline OmpE35 & $4.4 \pm 0.2$ & 1.42 & $3.1 \pm 1.3$ & $25.8 \pm 1.00$ & $-1.0 \pm 0.08$ \\
\hline OmpE36 & $3.0 \pm 0.2$ & 2.13 & $3.1 \pm 1.6$ & $22.3 \pm 0.64$ & $-1.5 \pm 0.04$ \\
\hline $\begin{array}{l}\text { OmpK3 } \\
5\end{array}$ & $4.4 \pm 0.4$ & 1.36 & $3.6 \pm 1.4$ & $14.9 \pm 0.4$ & $-0.6 \pm 0.06$ \\
\hline $\begin{array}{l}\text { OmpK3 } \\
6\end{array}$ & $2.5 \pm 0.2$ & 1.68 & $2.8 \pm 1.4$ & $19.1 \pm 0.36$ & $-1.2 \pm 0.09$ \\
\hline
\end{tabular}

Table 1 | OmpF/C orthologues physical properties. Measured conductivity and selectivity from single-channel electrophysiology (SI Materials and Methods); the average values from the molecular dynamics runs: the minimum radii in the $\mathrm{CR} /$, the average transversal electric field in the $\mathrm{CR}\left(\mathrm{E}_{\mathrm{p}}\right)$, and the average electrostatic potential in the $\mathrm{CR}\left(\mathrm{V}_{\mathrm{p}}\right)$.

\section{Experimental determination of permeability}

Liposome swelling assay (LSA) was selected to measure permeability of porins. The permeability of nine clinically relevant antibiotics (Fig.2, Supplementary Table 2) through eight distinct channels (Fig. 3) provides a dataset comprising 72 independent measurements. Each set of antibiotics is normalized with respect to glycine permeability for a given porin.

The LSA results for OmpF permeability are consistent with previous LSA data ${ }^{16}$ and with influx rate measurements in intact cells ${ }^{13-15}$. 

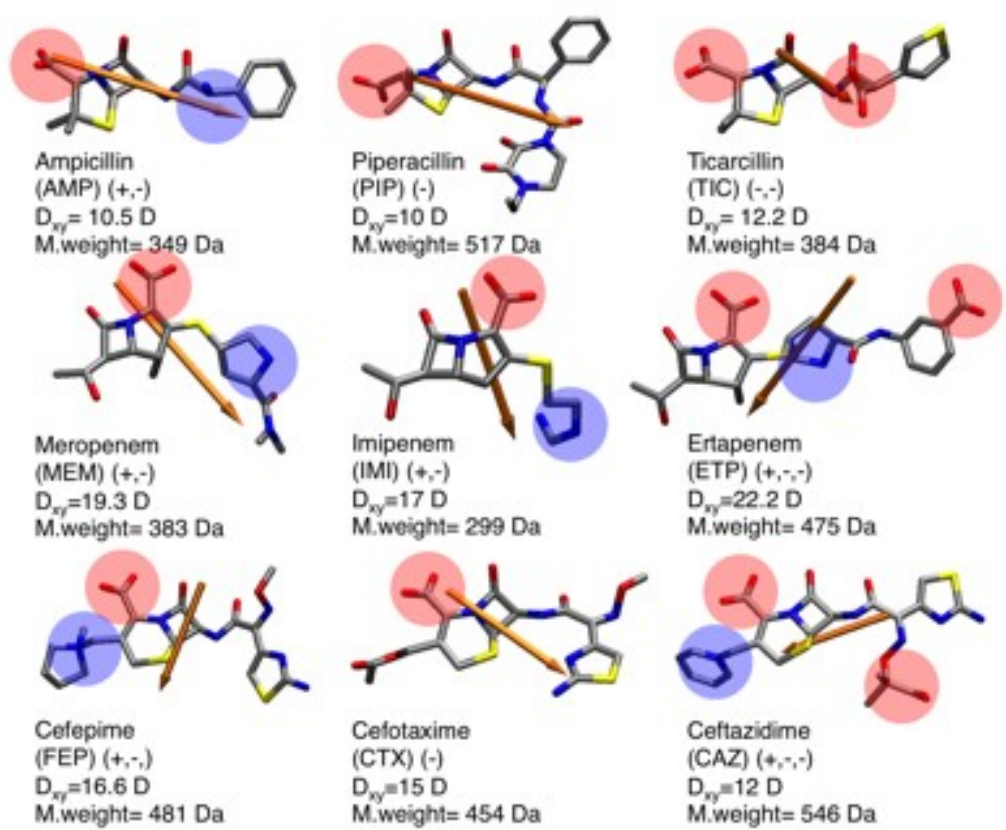

Figure 2 | Molecular properties of selected clinically relevant antibiotics. Molecular structure of the nine clinically relevant antibiotics used in the study. Each antibiotic is represented as a licorice model with its total dipole moment depicted as an orange arrow. Charged groups at $\mathrm{pH} 7$ are highlighted in red (negative) and blue (positive). The charged groups, the component of the dipole moment perpendicular to the long axis ( $D_{x y}$, in Debye) and the molecular weight are reported for each molecule.

As might be expected from the respective pore sizes (Table 1), molecules have a higher permeability through OmpF orthologues than through OmpC orthologues. The zwitterionic molecules, meropenem (MEM), imipenem (IMI), and cefepime (FEP), exhibited high relative permeabilities (40-80\% of glycine permeability) for all porins. Ampicillin (AMP) had lower relative permeability, resembling more the negatively charged molecules ceftazidime (CAZ), cefotaxime (CTX), piperacillin (PIP) and ticarcillin (TIC), which displayed permeabilities ranging from $0-40 \%$. In contrast, ertapenem (ETP), which also has a net negative charge, showed elevated relative permeability, similar to that of zwitterionic molecules, except for OmpE35 and OmpE36 (Fig. 3). Overall, MEM showed the highest relative permeability through porins ( $60 \%$ ) while PIP (larger size and negatively charged) and TIC (dianionic) had the lowest. 

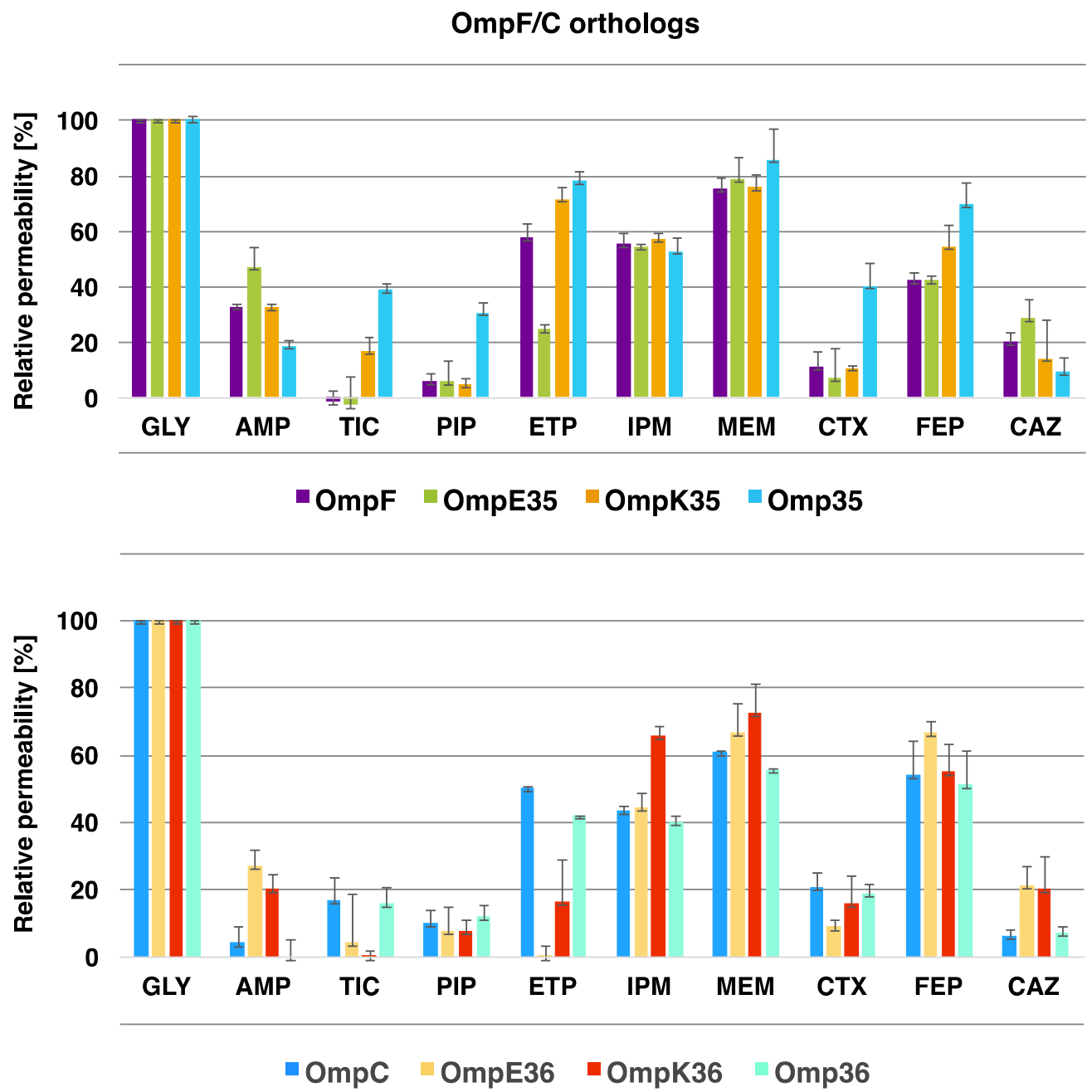

Figure 3 | Relative permeability of $\beta$-lactams through $\mathrm{OmpF} / \mathrm{C}$ orthologues. Permeation rates determined from LSA experiments. Data are displayed for the relative permeability of nine antibiotic molecules, e.g. ampicillin (AMP), ticarcillin (TIC), piperacillin (PIP), ertapenem (ETP), imipenem (IMP), meropenem (MEM), cefotaxime (CTX), cefepime (FEP) and ceftazidime (CAZ), through porins of E coli (OmpF and OmpC), E. aerogenes (Omp35 and Omp36), E. cloacae (OmpE35 and OmpE36) and $K$. pneumoniae (OmpK35 and OmpK36), normalized with respect to glycine (GLY), which is set to $100 \%$.

\section{The scoring function for permeability: connecting structure to transport}

In order to quantify antibiotic permeability through porins, we need a theoretical model for permeability to understand how the molecular-scale properties of porins control the macroscopic flow of molecules. If we treat the antibiotic translocation process as a one-dimensional diffusion-drift problem ${ }^{36,37}$, the molecular flux through the channel follows Fick's law, $J=-N_{a} P S_{0} \Delta c$, where $\Delta c$ is the difference of the antibiotic molar concentrations, $S_{0}$ is the geometrical cross section at the mouth of the channel and $\mathrm{N}_{\mathrm{a}}$ is the Avogadro constant. The permeability coefficient $\mathrm{P}$, reflects the ability of a certain molecule to translocate through the channel. Hence, its calculation requires the knowledge of the pore-molecule interaction energy along the whole length of the pore (Materials and Methods). This interaction energy, $\mathrm{U}(\mathrm{z})$ can be determined from all-atom MD simulations, but its determination is computationally 
expensive even when applying enhanced sampling techniques ${ }^{38}$, prohibiting virtual screening of large compound libraries. Nevertheless, as previously reported ${ }^{29}$ the main contribution to the pore-molecule interaction, $\mathrm{U}(\mathrm{Z})$, comes from the free-energy barrier located in the CR. Therefore, the permeability coefficient can be approximated to $P=\frac{D_{\text {eff }}}{\Delta L} e^{-\frac{1}{k_{B} T} U_{C R}}$, the free-energy barrier for the permeating molecule in the $\mathrm{CR}, \Delta \mathrm{L}$ the length of the $\mathrm{CR}, \mathrm{kB}$ the Boltzmann constant, and $\mathrm{T}$ the temperature. Within this approximation, the logarithm of the permeability coefficient can be directly connected to the freeenergy barrier in CR only ${ }^{29,37}, \ln (P)=-\frac{1}{k_{B} T} U_{C R}+$ const scoring function for permeability from the knowledge of a few accessible molecular parameters that can be directly calculated from MD (Materials and Methods: Scoring Function derivation and calculation protocol):

$$
S_{c}=\ln (P)=\alpha \cdot U_{\text {steric }}+\beta \cdot\left(Q_{m} \cdot V_{p}\right)+\gamma \cdot\left(D_{m} \cdot E_{p}\right)+\delta
$$

The first term accounts for the size exclusion problem inside the CR (Fig. 4), which depends on both the average size of the pore (minimum cross-section) and molecule (minimum projection area). This entropic term determines a barrier for molecules and controls spontaneous uptake. The second and the third terms of Eq.1 account for the electrostatic interactions of the diffusing molecule with the pore, through the electrostatic potential $\left(V_{p}\right)$ and electric field $\left(E_{p}\right)$, interacting respectively with the charge $\mathrm{Q}_{\mathrm{m}}$ and the dipole $\mathrm{D}_{\mathrm{m}}$ of the molecule. These two terms modulate the total barrier for permeation (Fig. 4) and can decrease the effect of the steric barrier. In our model the selectivity of these porins for cations arises from their interaction with the negative electric potential inside the CR. It should be noticed that although the mechanism is general, an entropic barrier modulated by electrostatic interactions, the same molecule will exhibit different permeability rates in different pores. Pore structural changes are reflected in changes in the minimum cross-section area and electrostatic properties that must be calculated from the MD simulation of the pore in a realistic environment.
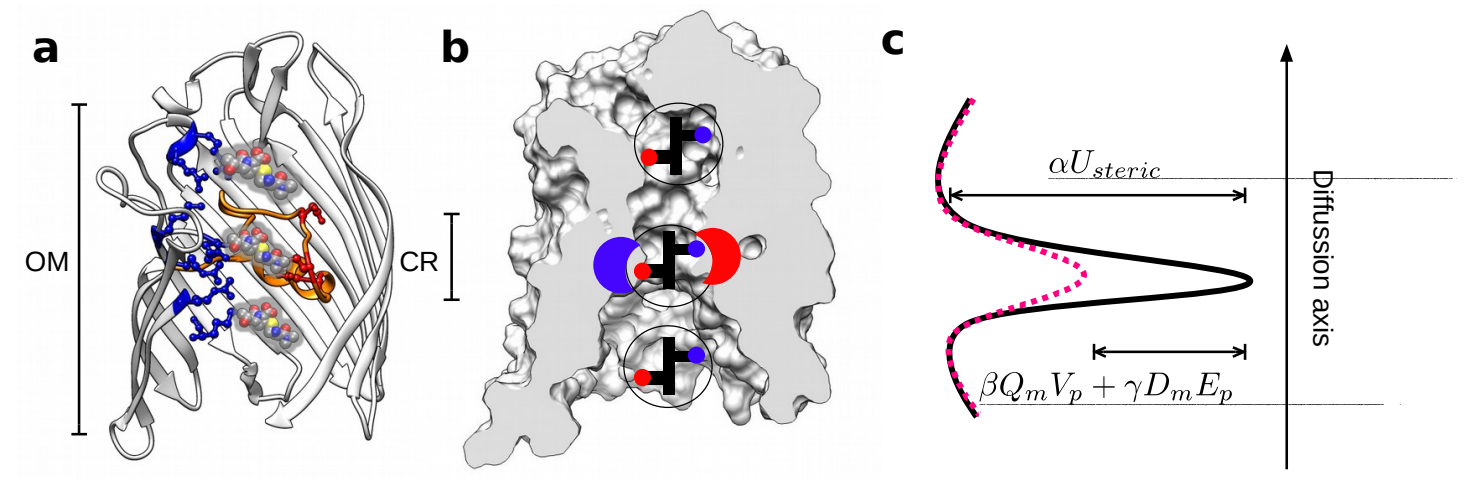

Figure 4 | Modulation of the total barrier for permeation. a, All-atom representation of the permeation of a small molecule through one OmpF (ribbons) monomer. b, Schematic representation of the relevant descriptors for the scoring function. The molecule is represented by its size (black oval) and charge distribution, while the porin is represented by its hourglass shape and charge distribution in the CR. c, Energetic decomposition of the total free-energy barrier faced by small molecules during permeation through porins. 
The standard LSA technique also assumes Fick's law for the substrate flux ${ }^{39}$, hence the total permeability of a substrate $a$ is proportional to the corresponding permeability coefficient. Because experimentally we have access to relative measurements: $P_{\exp }(a)=\frac{P(a)}{P(\text { glycine })}$, the shift $\delta$ in Eq.1 can be redefined including the permeability of glycine.

To obtain the parameters for the scoring function, Eq.1, we applied standard least square method using $\alpha, \beta, \gamma$, and $\delta$ as fitting parameters to reproduce the measured LSA data of Fig. 3. The results shows an excellent correlation, $r=0.86$, with a root mean square error (RMSE) of 0.47 (Fig. 5a). The analysis of the results grouped by charge (Fig. 5b), size (Fig. 5c) or transversal dipole moment of each molecule (Fig. 5d) allows several general observations. (i) Anionic molecules are disfavoured due to electrostatic repulsion by the porins, which to varying degrees are cation selective (Fig. 5.b). (ii) The size of the molecule, defined as its minimal projection area, correlates with permeability (Fig. 5c). (iii) The magnitude of the transverse dipole moment of a molecule $D_{\text {xy }}$ (with respect to the main inertia axis of the molecule) is directly correlated with permeability (Fig. 5d). The alignment of the transversal dipole moment of the molecule to the transversal electric field of the pore is always a favorable interaction (Eq.1), hence it decreases the barrier and increases molecular permeability. Although, from Fig. 5d the correlation of $D_{x y}$ looks weak when confronted with the charge or the size (Fig. 5b,c), in some cases, like ertapenem, it can compensate for the charge penalty imposed by the selectivity of the pore. Ertapenem has a net negative charge (Supplementary Table 2) and its size $\left(\sim 57 \AA^{2}\right)$ is comparable to that of the negatively charged cefotaxime $\left(\sim 59 \AA^{2}\right)$ and zwitterionic cefepime $\left(\sim 58 \AA^{2}\right)$. However, its permeability is similar to cefepime permeability and higher than that of cefotaxime, since its charge distribution shows the biggest transversal dipole moment ( 22.2 Debye, Supplementary Table 2) of the set of antibiotics considered. 
a

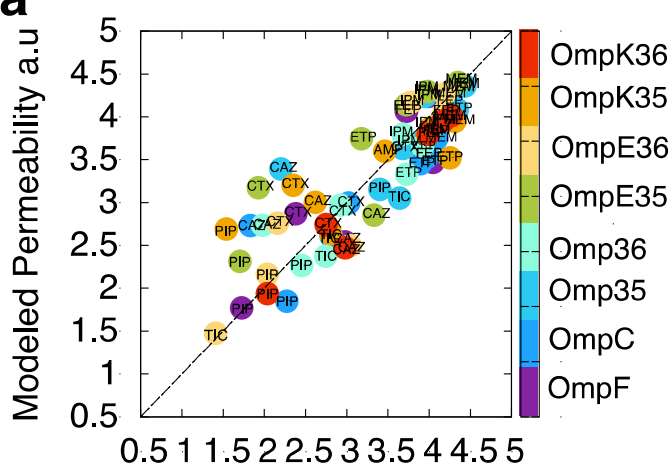

C $\operatorname{Ln}($ Experimental Permeability) a.u

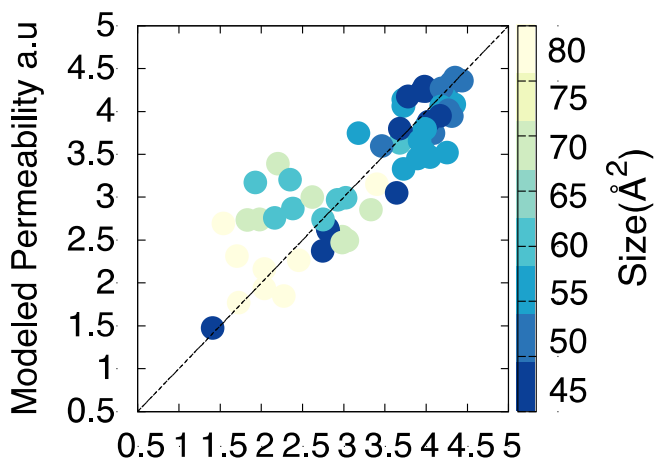

Ln(Experimental Permeability) a.u b

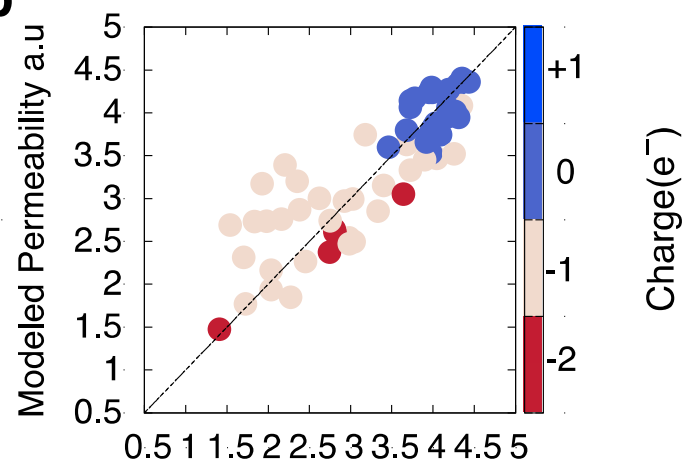

d

Ln(Experimental Permeability) a.u

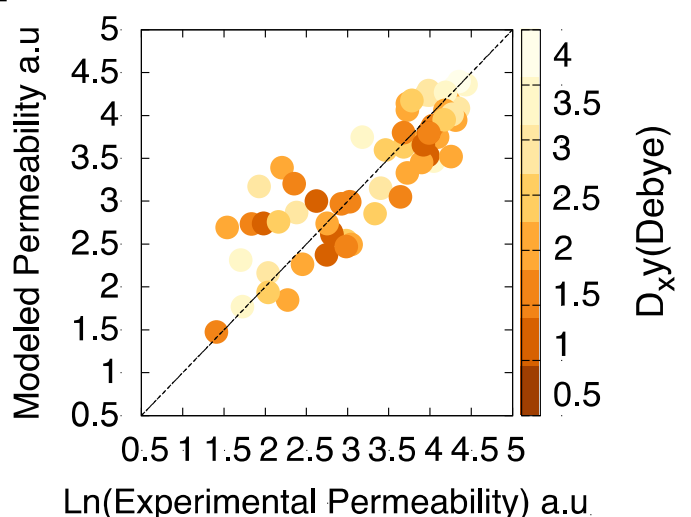

Figure 5 | Measured vs. predicted permeability of antibiotics through Gramnegative bacterial porins. Correlation between modelled permeability and relative permeability estimated in liposome swelling experiments. Modelled permeability vs. experimental permeability is shown with respect to porin and compounds identity: ampicillin (AMP), ticarcillin (TIC), piperacillin (PIP), ertapenem (ETP), imipenem (IMP), meropenem (MEM), cefotaxime (CTX), cefepime (FEP) and ceftazidime (CAZ), a, and with respect to several molecular properties, namely charge, b, molecule size, $\mathbf{c}$, and transversal dipole, $\mathbf{d}$.

To test the assumption that glycine has the same permeability in all pores, we include a shift parameter $\left(\delta_{\mathrm{i}}\right)$ and fit it separately for each pore. Moving from four to eleven fitting parameters, we obtained $r=0.88$, with a RMSE of 0.43 . This small variation supports our initial assumption.

While our data suggests that performing LSA experiments with the same system for charged and uncharged molecules gives reasonable results (Fig. 5), the use of LSA with charged molecules can in principle pose problems due to the movement of the respective counter ions ${ }^{16}$. We therefore cross-validated the scoring function for charged molecules using single-molecule electrophysiology data (Supplementary Table 3). Assuming the barrier to enter the current-blocking state is similar (or 'proportional') to the barrier for permeation, the logarithm of the measured association rate $\left(\log \left(k_{\text {on_ave }}\right)\right)$ can be correlated to the $\log (\mathrm{P})$. The correlation coefficient for scoring function (with the $\alpha, \beta, \gamma$, and $\delta$ previously fitted to LSA data) versus $\log \left(k_{\text {on_ave }}\right)$ is 0.75 (Supplementary Figure 6 ), validating LSA data for charged molecules.

Testing the scoring function on bacteria: $\beta$-Lactam activity and flux through porins 
In order to validate our scoring function and the LSA results with in vitro antibacterial data, the ability of $\beta$-lactams to cross the OM through the different porins was determined using time-kill assays. We selected the cephalosporins cefepime and ceftazidime, as readily and poorly permeating substrates respectively (Fig. 6). As expected ceftazidime exhibited faster killing on cells expressing OmpF-like ( $\sim 0.01 \%$ survival at $\mathrm{t}=150 \mathrm{~min}$, almost no survival at $\mathrm{t}=180 \mathrm{~min})$ than it did on cells expressing OmpC-like porins ( 1-10\% survival at $\mathrm{t}=150 \mathrm{~min}$ and $180 \mathrm{~min}$ ) ( Figure 6 , left panel). Cefepime, on the other hand, exhibited similar killing kinetics regardless the type of porin expressed $(\sim 0.001-0.1 \%$ survival at $t=150 \mathrm{~min}$, almost no survival at $\mathrm{t}=180 \mathrm{~min}$ ) (Figure 6, right panel). This is generally consistent with the scoring function prediction, where the zwitterionic cefepime is expected to have better permeability than for the anionic and larger ceftazidime.

The scoring function permeability predictions are also in agreement with results reported in literature $14,15,40$. For example, it correctly describes the increased permeability of ampicillin with increasing salt concentration in OmpC ${ }^{15}$ and not in OmpF (Supplementary Figure 7a,b). It is interesting to note that this behavior is common to all the considered zwitterionic molecules (Supplementary Figure 7a) due to the screening of the internal electric field of OmpF with increasing salt concentration ${ }^{28}$ while the OmpC internal electric field remains unaltered. The reason for this is that the water polarization-based method ${ }^{28}$, used to calculate the internal electric field of the channel, explicitly takes into account the media and hence its salt concentration. Conversely, this behavior is not observed for negatively charged molecules (Supplementary Figure 7c), where the screening of the electrostatic potential, upon ion addition, plays a larger role at low concentrations than the effect of the electric field. Further, the scoring function predicts a higher permeability for ampicillin through OmpF and OmpC, than benzylpenicillin at different salt concentrations (Supplementary Figure 7d). This result is in agreement with previous literature ${ }^{14}$ (Supplementary Figure 7d, black dashed line) and highlights the importance of the presence of a positive group in the scaffold ${ }^{40}$. The addition of a positive charge to the anionic benzylpenicillin to obtain ampicillin alters the electrostatic properties making ampicillin zwitterionic and increasing its dipole moment (from 18 Debye to 34 Debye) leading to increased permeability, although ampicillin $\left(\sim 50 \AA^{2}\right)$ has a larger minimal projection area than benzylpenicillin ( 46 $\left.\AA^{2}\right)$.

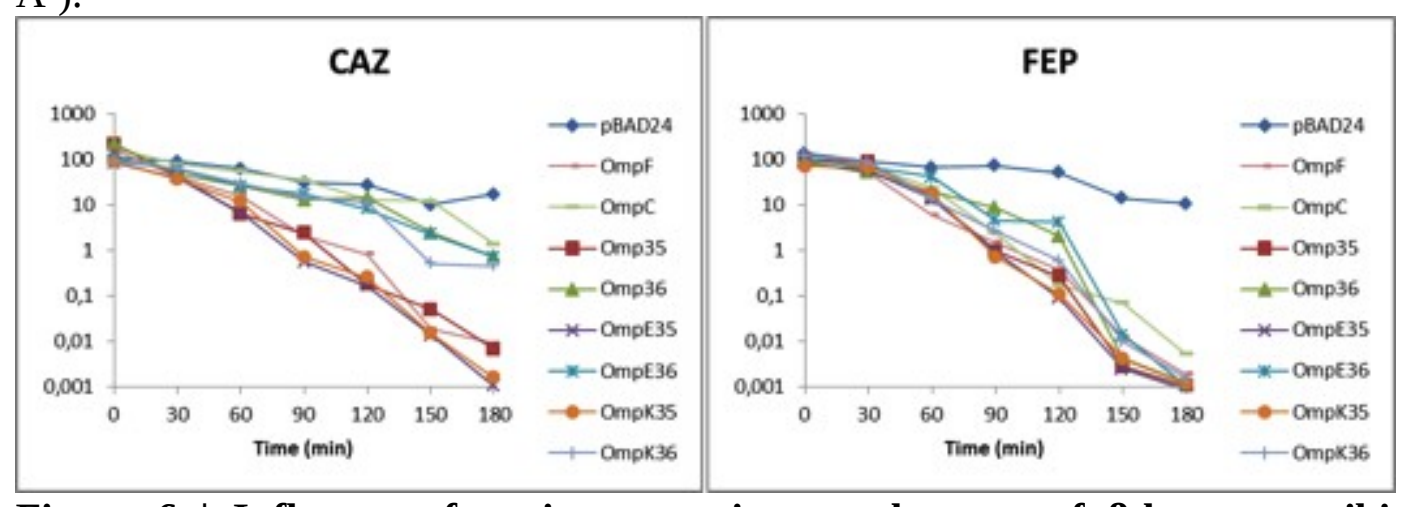

Figure 6 | Influence of porin expression on the rate of $\beta$-lactam antibiotic activity. Percentage decrease in $\mathrm{cfu} / \mathrm{ml}$ of $\mathrm{K} 12 \Delta \mathrm{FC}$ cultures expressing individual porin orthologues upon exposure to inhibitory concentrations of ceftazidime (CAZ $0.25 \mu \mathrm{g} / \mathrm{ml}$, left panel) or cefepime (FEP, $0.125 \mu \mathrm{g} / \mathrm{ml}$, right panel). Experiments were repeated at least three times.

Testing the scoring function on bacteria: non $\beta$-lactam scaffolds 
We also tested the performance of the scoring function predictivity with some non $\beta$ lactam molecules (not included in the training set), using the recently published whole-cell accumulation data ${ }^{10}$. Although, accumulation studies do not necessarily correlate directly with outer membrane permeability (e.g. due to inner membrane permeability or efflux), we selected a subset of molecules whose properties suggested that they were likely to enter the cell through porin-mediated transport (Supplementary Figure 8). The scoring function presented here was able to correctly describe changes in permeability caused by chemical substitutions that significantly altered charge, size, shape (linearity) and dipole moment (Supplementary Figure 8, Supplementary Table 4) and correlated with accumulation data ${ }^{10}$. Molecules with a net positive charge (1,2,3 in Supplementary Figure 8) showed higher accumulation and have better permeability according to our scoring function (Supplementary Figure 8) than their neutral versions (13,14,15 in Supplementary Figure 8). This is due to the favourable interaction of the net positive charge with the negative electrostatic potential in the CR. According to accumulation data ${ }^{10}$, molecule 19 is an example of how globularity affects negatively accumulation (Supplementary Figure 8, Supplementary Table 4). In our scoring function globularity is taken into account in terms of the minimal projection area. Molecule 19 has a high minimal projection area; hence we predict a low permeability (Supplementary Figure 8). Due to the same reason, molecule 3 is predicted to have a lower permeability than molecules 1 and 2, reproducing the trend of the accumulation data (Supplementary Figure 8, Supplementary Table 4). The correlation of the whole cell accumulation data with the predictions of the scoring function on molecules completely unrelated to the $\beta$ lactams suggests the potential utility of the scoring function despite the small size and $\beta$-lactam-focus of the training set.

\section{DISCUSSION}

General porins are the principal route of entry for polar antibiotics such as fluoroquinolones, penicillins, cephalosporins and carbapenems ${ }^{17-19}$ in Enterobacteriaceae. Previous understanding of molecular transport, based on electrophysiological measurements and simulations, identified the CR as the ratelimiting step for transport ${ }^{19,29}$. With the identification of new crystallographic structures from different porins of Enterobacteriaceae we established the key structural features of the CR (electric field, electrostatic potential and size) in general porins. While previous studies have been focused in the exploration of the chemical space of molecules only, here we included the exploration of porin diversity via the structural determinants. This has allowed us to analyze experimental transport data in term of pore-molecule interaction terms and validate the underlying molecular mechanism ${ }^{37}$. The proposed scoring function is based on well-defined physicochemical properties coming from main porin features: an entropic barrier, related to the hourglass shape of porins, modulated by the electrostatic interactions of the molecule in the charge-segregated CR of the porin. As it is designed, a compound is simply described by its partial atomic charges (to determine charge and dipole) and size (minimal projection area), hence, there is no dependence on specific chemical groups. This should allow general use of the scoring function as these parameters are straightforward to calculate, even if they are not as familiar as other descriptors such as molecular weight or clogP.

Our scoring function reproduces the measured permeability differences between two related molecules, benzylpenicillin and ampicillin ${ }^{14}$, confirming the importance of a 
positively-charged group in the scaffold to enhance permeation ${ }^{10}$ (Supplementary Figure 7d). Moreover, it correctly accounts for the modulation of ampicillin permeation through $\mathrm{OmpF}$ and $\mathrm{OmpC}$ as a function of media osmolarity ${ }^{15}$ (Supplementary Figure 7a,b), thanks to the water polarization-based method ${ }^{28}$ used to calculate the internal electric field of the channel, which explicitly takes into account the media and hence its salt concentration. The importance of the transversal component of the dipole moment with respect to the main axis of inertia of the molecule to aid permeation is also highlighted. As observed for ertapenem and ampicillin vs. benzylpenicillin, the introduction of a positive group in the scaffold increases permeability due to the increase of the transversal electric dipole moment.

Although as mentioned accumulation studies do not necessarily correlate directly with porin permeability, we were able to rationalize with a robust molecular mechanism (Fig. 4) the empirical rules proposed by Richter et al. ${ }^{10}$ to enhance accumulation into Gram-negative cells. The non-globularity of molecules required for accumulation is related to the size exclusion problem of the CR of these porins. This aspect is taken into account into the scoring function by using the minimal projection area of the molecule instead of the more widely used molecular weight ${ }^{7}$. Further, the observed higher accumulation conferred by addition of a primary amine ${ }^{10}$ is directly related to the intrinsic negative electrostatic potential calculated inside CR (Fig. 3g-i). This approach has been used to modify antibiotics to significantly increase their intracellular accumulation in Gram-negative bacteria ${ }^{10,40}$. However, it is notable that many marketed compounds that transit through porins ${ }^{41,42}$ are zwitterionic at physiological $\mathrm{pH}$. While this may lead to lower absolute accumulation ${ }^{43}$ inside bacteria, these antibiotics have been selected not only for optimum penetration (i.e. activity) balanced with appropriate drug-properties (pharmacokinetics, safety, etc.) ${ }^{7,43-45}$. Based on the results presented here, our model is able to accurately rank molecules in terms of their permeability thus making it feasible to use it to optimise potentially useful series of novel antibiotics.

Our approach offers a different perspective for predicting permeability through the $\mathrm{OM}$ of Gram-negative bacteria: instead of searching for an energy minimum or a binding site obtained by docking procedures, it assesses how a molecule can compensate the inherent entropic barrier of pores. This approach provides a set of molecular determinants that are easily calculated: size, dipole moment and net charge. The molecular mechanism only depends on porin structural features, hence the derived scoring function can be readily extended to porin variants occurring in resistant strains selected, for example, by exposure to carbapenems, to provide molecular insights into the development of resistance ${ }^{19}$. Moreover, this procedure is computationally efficient and can potentially be used to screen large virtual libraries to identify new scaffolds with good permeation through porins. This approach will allow computational approach to improve antibiotics with poor porin permeation and define scaffolds with optimal porin permeability that might be used as starting point for developing new antibiotics. 


\section{METHODS}

X-Ray Crystal Structure Determination of $E$. cloacae OmpE35, OmpE36, $K$. pneumoniae OmpK35, OmpK36 and E. aerogenes Omp35 and Omp36

Genomic DNA from E. cloacae (ATCC 13047) and K. pneumoniae (ATCC 43816) was obtained from Basilea Pharmaceutica International Ltd. The genes including the signal sequence was amplified using PCR with the following primers:

OmpE35_for 5'-GCGCCATGGGCATGAAGCGCAATATTCTG-3',

OmpE35_rev 5'-CTAGTCTAGATTAGAACTGGTAAACCAGAC-3',

OmpE36_for 5'-GCGCCATGGGCAAAGTTAAAGTACTGTC-3',

OmpE36_rev 5'- CTAGTCTAGATTAGAACTGGTAAACCAGAC-3',

OmpK36_for 5'-GCGCCATGGGCAAAGTTAAAGTACTGTC-3',

OmpK36_rev 5'-CTAGTCTAGATTAGAACTGGTAAACCAGGC-3',

OmpK35_for 5'-CTAGGAATTCACCATGATGAAGCGCAATATTCTGGCA-3',

OmpK35_rev 5'-CTAGTCTAGATTAGAACTGGTAAACGATACCCACG-3'.

The PCR products were digested with NcoI and XbaI or EcoRI and XbaI for ompK35, and ligated with the pBAD24 $4^{46}$ or pB22 vector ${ }^{47}$ for ompK35 under the control the arabinose-inducible promoter. The ligated product was electroporated into $\mathrm{DH} 5 \alpha$ competent cells and plated on LB-ampicillin plates $(100 \mu \mathrm{g} / \mathrm{mL})$ for overnight incubation at $37^{\circ} \mathrm{C}$. Screening for positive clones was done using colony PCR, and clones were confirmed by DNA sequencing (Eurofins MWG). Porin-deficient E. coli BL21 (DE3) Omp8 competent cells ( $\triangle$ lambB ompF::Tn5 $\triangle$ ompA $\triangle$ ompC) ${ }^{48}$ were transformed with one of the positive clones, and protein was expressed using $0.1 \%$ arabinose for induction $\left(37^{\circ} \mathrm{C}, 3 \mathrm{~h}\right)$. The cells were harvested by centrifugation [1,914 × g for 30 min (Avanti J-26 XP Centrifuge, Beckman Coulter Inc.)] and lysed with a cell disrupter $(0.75 \mathrm{~kW}$; Constant Systems; one pass at $23 \mathrm{kpsi})$. Total membranes were collected by ultracentrifugation using a $45 \mathrm{Ti}$ rotor (Beckman Coulter Inc.; $45 \mathrm{~min}$; 42,000 rpm). The resulting membrane pellet was extracted twice with $0.5 \%$ N-lauroylsarcosine (sarkosyl) detergent (in $20 \mathrm{mM}$ HEPES, pH 7.5) followed by ultracentrifugation using a 45 Ti rotor (Beckman; 30 min, 42,000 rpm) to solubilise and remove inner-membrane proteins, followed by an overnight extraction at $4{ }^{\circ} \mathrm{C}$ in $1 \%$ lauryldimethylamine-oxide (LDAO) in $10 \mathrm{mM}$ HEPES, $50 \mathrm{mM} \mathrm{NaCl}$ (pH 7.5) to solubilise the OMPs (OMP extraction for OmpK35 was done using 3\% eluent for only one hour unlike the rest). LDAO extraction was followed by ultracentrifugation using a $70 \mathrm{Ti}$ rotor (Beckman; $30 \mathrm{~min}, 50,000 \mathrm{rpm}$ ) and the supernatant was subjected to Resource $\mathrm{Q}$ anion-exchange chromatography in $0.2 \%$ LDAO at $\mathrm{pH} 7.5$ (10 mM HEPES, $50 \mathrm{mM} \mathrm{NaCl})$. After elution, the protein was further purified by gel-filtration chromatography in $10 \mathrm{mM}$ HEPES, $100 \mathrm{mM} \mathrm{LiCl}$, 0.4\% C8E4 (pH 7.5). The purified protein was concentrated and crystal trays were set up at $10 \mathrm{mg} / \mathrm{mL}$. The crystal hits obtained in each case were optimised to obtain good-quality crystals (see Supplementary Table 5). Crystals were harvested, cryoprotected, harvested with glycerol ( 20\%), and flash- frozen in liquid nitrogen. X-ray diffraction data were collected at the Diamond Light Source and processed using $\mathrm{XDS}^{49}$. The crystal structure was solved by molecular replacement using MolRep $^{50}$ with following search models, E. coli OmpC with PDB code 2J1N (for OmpE36), K. pneumoniae OmpK36 with PDB code 1OSM (for OmpK36), E. coli OmpF with PDB code 2OMF (for OmpE35) and E. coli OmpF with PDB code 4GCP (for OmpK35). The refinement was done in Refmac5 ${ }^{50}$, and $\operatorname{Coot}^{51}$ was used for model (re) building. 
Codon-optimized genes encoding for Omp35 (ATCC 15038) and Omp36 (ATCC 13048) E. aerogenes were ordered from DNA2.0 (California, USA) and cloned into a pTAMAHisTEV ampicillin-resistant vector and transformed in porin-deficient $E$. coli BL21 (DE3) Omp8 $250 \mathrm{ml}$ of cell culture in LB containing $100 \mu \mathrm{g} \mathrm{ml}^{-1}$ ampicillin was incubated at $37^{\circ} \mathrm{C}$ and shacked at $200 \mathrm{RPM}$ overnight. $10 \mathrm{ml}$ of the overnight culture were used to inoculated 12x $1 \mathrm{~L} \mathrm{LB}$ containing the same amount of ampicillin and grown at $37{ }^{\circ} \mathrm{C}$ until $\mathrm{OD}_{600} \sim 0.6$. Cells were then induced with $0.4 \mathrm{mM}$ isopropyl $\beta$ D-thiogalactopyranoside (IPTG) and the temperature decreased to $25{ }^{\circ} \mathrm{C}$. The cells were let to grow 16 hours and then harvested by centrifugation at 6,200 g (JLA 8.1000 rotor, Beckman Coulter). Cell pellets were re-suspended in lysis buffer; 50 $\mathrm{mM} \mathrm{Na}_{3} \mathrm{PO}_{4} \mathrm{pH}$ 7.4, $250 \mathrm{mM} \mathrm{NaCl}$ 10\% (v/v) glycerol, $20 \mu \mathrm{g} \mathrm{ml}^{-1}$ DNAse, $100 \mu \mathrm{g} \mathrm{ml}^{-}$ ${ }^{1}$ lysozyme (both Sigma-Aldrich) and EDTA-free protease inhibitor cocktail (Roche). Cells were lysed by two passes through a chilled cell disruptor at $30 \mathrm{kpsi}$. Cellular debris was removed by centrifugation at 10,000 g (JA 25.50 rotor, Beckman Coulter). The total membrane fraction was collected by ultracentrifugation at 100,000 g (50.2 Ti rotor, Beckman Coulter) at $4{ }^{\circ} \mathrm{C}$ for 1 hour. The membrane was solubilized with $7 \%(\mathrm{v} / \mathrm{v})$ Octyl-POE and left stirring at $4^{\circ} \mathrm{C}$ overnight. Soluble material was then recovered by ultracentrifugation at $4{ }^{\circ} \mathrm{C}$ for 1 hour. The supernatant containing the solubilized protein was incubated with $5 \mathrm{ml}$ of Ni-NTA resin (Qiagen, UK) and 10 $\mathrm{mM}$ imidazole $\mathrm{pH} 8$ at $4{ }^{\circ} \mathrm{C}$ for 2 hours. The resin was then washed with $10 \mathrm{CV}$ of washing buffer; $50 \mathrm{mM} \mathrm{Na} \mathrm{PO}_{4} \mathrm{pH}$ 8, $250 \mathrm{mM} \mathrm{NaCl}, 1 \%$ (v/v) Octyl-POE (Bachem) and $30 \mathrm{mM}$ imidazole $\mathrm{pH}$ 8. The protein was eluted with the same buffer containing $250 \mathrm{mM}$ imidazole. Consecutively, $1 \mathrm{mg}$ of $\mathrm{His}_{6}$-tagged TEV protease was added to the eluted protein and dialyzed in SnakeSkin tubing (Thermo Scientific) against a buffer containing $10 \mathrm{mM}$ imidazole at $4{ }^{\circ} \mathrm{C}$ overnight. The dialyzed sample was passed through a $0.45 \mu \mathrm{m}$ syringe filter and applied to a $5 \mathrm{ml}$ His-trap column (GE Healthcare). The cleaved protein was collected from the flow-through fraction and further purified by gel filtration using a 16/ 60 Superdex 200 pg (GE Healthcare) equilibrated with $2 \mathrm{CV}$ of a buffer containing $10 \mathrm{mM}$ Tris- $\mathrm{HCl}, 150 \mathrm{mM} \mathrm{NaCl}$ and $0.45 \%(\mathrm{w} / \mathrm{v}) \mathrm{C}_{8} \mathrm{E}_{4}$ (Bachem). The protein was concentrated to $10 \mathrm{mg} \mathrm{ml}^{-1}$ for crystallization. Purity and integrity were monitored on SDS-PAGE (NuPAGE, Invitrogen) and mass spectrometry. Different commercial screens were used for initial trials. When required hits were optimized by hanging-drop vapor diffusion technique. Crystals were harvested and cool flashed in liquid nitrogen. Ethylene glycol was frequently used as cryoprotectant. Listed below are the crystallization conditions that gave the crystals used for data collection. Data were collected at the Synchrotron Light Source and processed using Miosflm ${ }^{52}$ and scaled with SCALA ${ }^{53}$. MR Phaser ${ }^{54}$ from the Ccp4 suit was used to solve the structures. OmpK36 (ID PDB: 1OSM) was used as search model to solve Omp36 structure, while OmpF (ID PDB: 2OMF) was used as search model to solve Omp35. The structures were built manually using $\operatorname{Coot}^{51}$ and refined using Refmac ${ }^{50}$. Data quality was checked with Molprobity ${ }^{55}$. The data collection and refinement statistics for all the proteins are summarised in Supplementary Table 6.

\section{Selectivity and conductance measurements}

The method of multi-channel measurements for the selectivity characterisation has been described in detailed in previous publication ${ }^{38,56}$. Briefly, a Teflon chamber composes the system with two compartments separated by a thin wall. A circular hole with an area of $1 \mathrm{~mm}^{2}$ located on this wall were painted by $1 \%(\mathrm{w} / \mathrm{v}) \mathrm{DPhPC}$ dissolved in n-decane. Membrane formation was monitored under a microscope with 
light reflection, which turned optically black gradually indicating the bilayer formation.

In all the experiments 1,2-diphytanoly-snglycero-3-phosphocholine (DPhPC) from Avanti polar lipids, Inc. was used. Chloroform, 4-(2-hydroxyethyl)-1piperazineethanesulfonic acid (HEPES), and Potassium hydroxide (KOH) were obtained from Carl Roth GmbH. Potassium chloride (KCl) and 2-(N-morpholino) ethanesulfonic acid (MES) polyamine spermine were obtained from Sigma-Aldrich. Octyl-Polyethylene glycol octyl ether (Octyl-POE) was obtained from Enzo Life Science.

The actual selectivity was recorded by application of a tenfold gradient in $\mathrm{KCl}$ alone ( 0.1 vs. $1 \mathrm{M} \mathrm{KCl}$ ). The zero current voltage was recorded using standard $\mathrm{Ag} / \mathrm{AgCl}$ electrodes with a salt bridge.

The methods of single channel measurements were carried out on the MECA (Multielectrode cavity array) chips (Ionera Technologies GmbH) using DPhPC as previously described by Baaken G. et al. ${ }^{57} .16$ cavities each with diameter $\varnothing 50 \mu \mathrm{m}$ are located on the MECA chip. Each cavity was painted automatically to form individual bilayers. The cavities are connected to the headstage of amplifier (Axon 200B, Molecular Devices) from the bottom (trans) and face a common bath solution, which is connected to the ground (cis). Briefly, $0.3 \mu \mathrm{L}$ of $0.1 \mathrm{mg} / \mathrm{ml} \mathrm{DPhPC} \mathrm{lipids} \mathrm{dissolved}$ in octane were added onto the MECA chip mounted in Orbit 16 (Nanion Technologies $\mathrm{GmbH}$ ), without pre-treating the cavities with additional solvent. The planar lipid bilayers were formed in an automated fashion by controlling a Teflon coated magnetic bar, this process was computer controlled by the OrbitControl software (Nanion Technologies GmbH).

After successful single channel reconstitution, the cis side of the chamber was manually perfused carefully to remove extra proteins in order to prevent further protein insertions. Single channel current measurements were digitized by a Digidata 1440 digitizer (Molecular Devices) and amplified by an Axopatch 200B amplifier (Molecular Devices) recorded in the voltage clamp mode through PClamp software (Molecular Devices). Signals were filtered by a low-pass Bessel filter at $10 \mathrm{kHz}$ acquired with a sampling rate at $50 \mathrm{kHz}$. The current amplitude and noise analysis were performed using Clampfit 10.8 (Molecular Devices). The measured parameters were the frequency of current transient spikes $\left(\mathrm{v}\left(\mathrm{s}^{-1}\right)\right)$ and averaged residence time $(\tau)$. The averaged residence time was calculated from exponential fit of total residence time measured from each transient spike recorded over the entire time scale. External transmembrane potentials were applied from the trans side, continuous constant voltages were applied for at least 60 sec to collect sufficient stochastic events. Hidden Markov Model was applied to investigate the substrate-protein interaction, which is considered as a one-binding site-two-barrier model. When same substrates were added from cis side only, two kinetic rates were obtained, i.e. association rate $\left(k_{\text {on }}\right)$ and dissociation rate $\left(k_{\text {off }}\right)$. The association rate, $k_{o n}$, is calculated from the current transient spike frequency, $k_{o n}=\frac{v}{3[c]}$, where [c] is the concentration at cis side. It is independent to the substrate concentration and determines the flux through each monomer (porins are composed with three identical monomers) when saturation does not occur. The dissociation rate, $k_{\text {off }}=\frac{1}{\tau}$, is inversely proportional to the residence time $(\tau)$. 


\section{Antibiotic list}

Carbapenems including ertapenem (ETP), meropenem (MEM) and imipenem (IPM) were purchased from Sequoia Research Products Ltd. (United Kingdom); cephalosporins including ceftazidime (CAZ), cefepime (FEP), cefotaxime (CTX) and penicillins including piperacillin (PIP) and ticarcillin (TIC) were from Sigma.

\section{Liposome swelling assay}

The liposome suspension mixture was prepared by mixing $100 \mathrm{mg}$ egg phosphatidycholine (solubilized in $25 \mathrm{mg} / \mathrm{ml}$ in chloroform; Avanti Polar Lipids) and $2.3 \mathrm{mg}$ dihexadecyl phosphate (dissolved in $1 \mathrm{ml}$ of chloroform). For each protein, $100 \mu \mathrm{l}$ from the liposome suspension was aliquoted in glass tubes and vacuum dried for 2 hours. The thin dried lipid layer was then solubilized in $100 \mu \mathrm{l}$ water along with the addition of required protein amount, such that all proteins have the same molar amount in each experiment set-up. This mixture was sonicated for 2 min before leaving for drying overnight in a desiccator. The control liposome mixture was prepared by adding buffer instead of protein into the liposome suspension. Next day, $200 \mathrm{ml}$ of $12 \mathrm{mM}$ of stachyose solution (in $10 \mathrm{mM}$ HEPES, pH 7.0) was added to the overnight dried proteolipid film and mixed gently before proceeding to the swelling assay. For each assay, $5 \mu \mathrm{l}$ of proteoliposome mixture was added to $100 \mu$ l of substrate solution.

The substrate concentration for each compound was determined empirically, so that no changes in optical density occur when measured with the control protein-free liposomes. Substrate addition was followed by rapid mixing before monitoring the optical density at $400 \mathrm{~nm}$ for $60 \mathrm{sec}$ at a $5 \mathrm{sec}$ interval (dead time experiment: $\sim 5 \mathrm{~s}$ ). The swelling assay rate for glycine permeation through OmpF of E. coli was taken as 100\% (as reference) for calculating the other permeation rates. To ensure equimolar amounts of proteins, $15 \mu \mathrm{g}$ for a protein with the molecular weight of $25 \mathrm{kDa}$ was set as the standard to calculate the amounts of each protein needed for the assays. The concentrations used were: glycine 10-12 mM, glucose 10-12 mM, arginine $8 \mathrm{mM}$, glutamate $10 \mathrm{mM}$, ampicillin $8 \mathrm{mM}$, meropenem 8-9 mM, imipenem 9-10 $\mathrm{mM}$, ceftazidime $10 \mathrm{mM}$, ertapenem $12 \mathrm{mM}$, cefepime $2 \mathrm{mM}$, cefotaxime $9-10 \mathrm{mM}$, ticarcillin $8 \mathrm{mM}$, piperacillin $10 \mathrm{mM}$. The empirically determined concentrations of certain substrates changed slightly for different liposome preparations.

\section{Molecular dynamics}

All 6 X-ray structures (Omp35, Omp36, OmpE35, OmpE36, OmpK35, OmpK36) were used as starting coordinates for molecular dynamics (MD) simulations. All the amino acid residues were simulated in their ionization state at neutral $\mathrm{pH}$ except for E296 (OmpF), D299 (OmpC), E102 (Omp35), D307 (Omp36), E102 (OmpK35), D297 (OmpK36), which were protonated (net charge 0), in all the three monomers for each trimmer, as suggested for OmpF by Varma et al. ${ }^{58}$.

For each porin, the entire trimer was embedded in a pre-equilibrated POPC (1palmitoyl-2-oleoyl-sn-glycero-3-phosphocholine) bilayer of 259 lipids and the system was oriented in order to center the protein at the origin of the coordinate system and align the channel along the $\mathrm{z}$-axis (positive $\mathrm{z}$ : extracellular side; negative $\mathrm{z}$ : periplasmic side). Each system was solvated in a $200 \mathrm{mM} \mathrm{KCl}$ solution. Only the last 
600 ns were used for the analysis

After 1 ps of energy minimization (conjugate gradients), a slow heating from 10 to $300 \mathrm{~K}$ was carried out for $1 \mathrm{~ns}$. During this stage, positional restraints were applied on the protein c $\alpha$-carbons (along all three dimensions), as well as on the lipids phosphorus atoms (along $\mathrm{z}$ only). After releasing the constraints on the POPC, an equilibration stage follows for 4 ns in the NPT ensemble at 1.0 bar and $300 \mathrm{~K}$. Finally, 700 ns MD simulations were performed in the NVT ensemble after the elimination of the protein restraints.

The NPT equilibration was performed with the program NAMD ${ }^{59}$, with 1.0 fs timestep, and treating long-range electrostatics with the soft particle mesh Ewald (SPME) method (64 grid points and order 4 with direct cutoff at $1.0 \mathrm{~nm}$ and $1.0 \AA$ grid-size). Pressure control was applied using the Nose-Hoover method (extended Lagrangian) with isotropic cell, integrated with the Langevin Dynamics (200 fs and $100 \mathrm{fs}$ of piston period and decay, respectively). The latter was also applied for temperature control with 200 fs thermostat damping time. Production runs in the NVT ensemble were performed with the ACEMD ${ }^{60}$ code compiled for GPUs, by rescaling hydrogen mass to 4 au and increasing the time-step up to 4.0 fs. The Langevin thermostat was used with 1 ps damping time. SPME was used to treat the electrostatics as for the equilibration stage. The Amber99SB-ILDN force field was used for the protein and lipids, and the TIP3P for waters. For all the known-antibiotics the Gaff-force field parameters where obtained from http://www.dsf.unica.it/ gmalloci/abdb/. All molecular properties where calculated from a $1 \mu$ s trajectory of the molecule in a $0.1 \mathrm{M} \mathrm{KCl}$ solution ${ }^{61}$. In the case of the three primary amines and their derivatives ${ }^{10}$ we used the ChemAxon's Marvin suite of programs ${ }^{62}$ to draw the structure and calculate the dominant tautomer distribution for each molecule and thus find the protonation/charge states most populated at physiological $\mathrm{pH}=7.4$. Atomic partial charges were then generated through the two-step restrained electrostatic potential (RESP) method ${ }^{63}$ implemented in the Antechamber package ${ }^{64}$.

\section{Scoring function: derivation and calculation protocol}

The antibiotic translocation process can be treated as a one-dimensional diffusiondrift problem ${ }^{36,38}$. Under this assumption, the channel permeability coefficient reads:

$$
P=\left[\int_{0}^{L} \frac{\exp \left(U(Z) / k_{B} T\right)}{D(Z)} d Z\right]^{-1}
$$

where $\mathrm{U}(\mathrm{Z})$ is the potential of mean force and $\mathrm{D}(\mathrm{Z})$ is the diffusion coefficient along the pore axis $(\mathrm{Z})$; $\mathrm{L}$ is the length of the pore and $\mathrm{k}_{\mathrm{B}} \mathrm{T}$ is the thermodynamic temperature. Although the diffusion model of Eq. 1 is governed by an integral of the free-energy profile $\mathrm{U}(\mathrm{Z})$ along the whole length $\mathrm{L}$ of the pore, the largest contribution comes from the free-energy barrier region, i.e. the CR. Hence, the integral in Eq. 1 can be approximated to the value of the integrand in the CR only, multiplied by, the length of the $\mathrm{CR}(\Delta \mathrm{L})$. Therefore, the simplified equation for the permeability reads:

$$
P=\frac{D_{\text {eff }}}{\Delta L} e^{-\frac{1}{k_{B} T} U_{C R}}
$$

where $D_{\text {eff }}$ is the effective diffusion constant in the CR and $U_{C R}$ is the free-energy barrier for the substrate in the CR. Within this approximation, the logarithm of the permeability coefficient can be directly connected to the free-energy barrier in CR, 
$\ln (P)=-\frac{1}{k_{B} T} U_{C R}+\ln \left(\frac{D_{\text {eff }}}{\Delta L}\right)$

Due to, (i) the weak logarithmic dependence of $\ln (\mathrm{P})$ on $\mathrm{D}_{\text {eff }}$ and $\Delta \mathrm{L}$, and (ii) the expected small variation of the two parameters, we can assume that the second term is the same for all substrates in all pores. The latter assumption is due to the fact that the length of the $\mathrm{CR}(\Delta \mathrm{L})$ is almost the same for all the pores considered. Moreover, the diffusion constant depends on the viscosity of the media and the radius of molecules (Supplementary Table 2), which does not vary more than $15 \%$ for small molecules. Hence,

$$
\ln (P)=-\frac{1}{k_{B} T} U_{C R}+\text { const }
$$

All the parameters included in the scoring function (Eq.1) were calculated from allatom MD simulations. The geometric cross-section area was calculated superimposing a grid onto each monomer of the trimmers and mapping for each frame all the protein atoms with their respective van-der-Waals radii. For each $\mathrm{Z}$ value, we sum the number of empty points of the grid to obtain the cross-section area at that particular $\mathrm{Z}$ and time. The internal electric field was calculated following the protocol in ${ }^{28}$. In the scoring function only the block average of the electric field in the CR is considered. The electrostatic potential was calculated by integrating the internal electric field along the waters putative path along the diffusion axis of the channel; its block average value in the CR is used for the scoring function.

Molecular properties were also calculated from all-atom MD trajectories of the molecule in a $0.1 \mathrm{MKCl}$ solution. Minimal projection areas were taken from http://www.dsf.unica.it/ gmalloci/abdb for the available molecules. For the new molecules from ${ }^{10}$, the minimal projection area was calculated with the molecular descriptors plugin of Marvin ${ }^{62}$. All dipole moments were calculated with Dipole Moment Watcher plugin of VMD ${ }^{65}$.

In order to test the dependence of the permeability results on the different terms scoring function we repeated the fitting procedure, using different combinations of the three terms of Eq.1, (Supplementary Figure 4). The entropic term ( $\left.U_{\text {steric }}\right)$ only, correlates poorly with the measured permeability ( $r=0.55$, Supplementary Figure $4 a)$, as does the size difference, $S_{m}-S_{p}$ (Supplementary Figure 4b, $r=0.59$, RMSE=0.72). Considering only the electrostatic potential term gives a higher correlation than either size difference or entropic term ( $\mathrm{r}=0.7$ Supplementary Figure 4c). Regarding the use of the electric field term, a hypothetical perfectly aligned molecule in the CR using the averaged total dipole of the molecule $\mathrm{D}_{\mathrm{m}} \mathrm{E}_{\mathrm{p}}{ }^{\mathrm{t}}$, yields a weak correlation $(\mathrm{r}=0.15$, Supplementary Figure $4 \mathrm{~d}$ ). However, if the transverse component to the main axis of inertia of the molecule is considered the correlation improves $(r=0.56, \mathrm{RMSE}=0.74$, Supplementary Figure 4e). Combining the two electrostatic terms we obtain a correlation of $r=0.81$ (RMSE $=0.52$ ) (Supplementary Figure $4 \mathrm{f}$ ). The combination of the first three terms $\left(U_{\text {steric }}+Q_{m} V_{p}+D_{x y}{ }^{m} E_{p}{ }^{t}\right)$ plus the constant shift $(\delta)$ gives a very reliable correlation coefficient $r=0.86$ (RMSE=0.47). On the other hand, using a different shift term for each pore, which implies that glycine can have a different permeability through the different pores, does not significantly improve the correlation coefficient ( $r=0.88$, RMSE=0.44), supporting our initial assumption.

Antibacterial activity and whole-cells-based assays to evaluate $\beta$-lactam flux through porins. 
Cultures of E. coli $\mathrm{K} 12 \Delta F C$ expressing the different porin orthologues were challenged with cefepime or ceftazidime at a fixed concentration that equals $2 \times$ MIC for the hypersusceptible wild-type K12 (i.e. cefepime, MIC of $0.06 \mu \mathrm{g} / \mathrm{ml}$; ceftazidime, MIC of $0.125 \mu \mathrm{g} / \mathrm{ml}$ ). CFU counts were determined by plating on $\mathrm{MH}$ agar and plotted as mean values of \% CFU decrease in the presence and absence of antibiotics over time (Supplementary Figure 7). All assays were performed in E. coli K12 strain W3110 and derivative porin mutants of (i.e. $\triangle o m p C, \triangle o m p F$ and $\Delta o m p C \Delta o m p F$, herein referred to as $\mathrm{K} 12 \Delta C$, $\mathrm{K} 12 \Delta F$ and $\mathrm{K} 12 \Delta F C$ ). The role of $E$. coli OmpF and OmpC was assayed in $\mathrm{K} 12 \Delta C$, $\mathrm{K} 12 \Delta F$ and in $\mathrm{K} 12 \Delta F C$ as a control porin-less strain. The role of OmpF and OmpC orthologs was investigated in $\mathrm{K} 12 \triangle F C$ transformed with either the empty vectors pBAD24 or pB22 or the recombinant plasmids containing E. aerogenes omp35, omp36 (pBAD24-omp35, pBAD24-omp36); E. cloacae ompE35, ompE36 (pBAD24-ompE35, pBAD24ompE36); and K. pneumoniae (pB22-ompK35, pBAD24-ompK36). Strains containing pBAD24 or pB22 derivative plasmids were cultured in Mueller Hinton II Broth (MHIIB) in the presence of ampicillin $(100 \mu \mathrm{g} / \mathrm{ml})$ and protein expression was induced with $0.1 \%$ L-arabinose (Sigma) for $2 \mathrm{~h}$ at $37^{\circ} \mathrm{C}$. Of note, E. coli $\mathrm{K} 12$ was used instead BL21 because it expresses the arabinose transporter AraE that ensures uniform uptake of arabinose and expression of proteins cloned under the control of an arabinose-inducible promoter. When tested for $\beta$-lactam permeability, cultures were diluted to $10^{7}$ cells $/ \mathrm{ml}$ in fresh MHIIB supplemented with $0.1 \%$ L-arabinose, $\beta$ lactamase inhibitors tazobactam and clavulanic acid $(4 \mu \mathrm{g} / \mathrm{ml}$ each) to inhibit the activity of the plasmid-borne $\mathrm{AmpC}^{66}$, and in the presence or in the absence of antibiotics. Minimum inhibitory concentrations (MICs) were determined for the K12 wild-type and K12 $\triangle F C$ strains by using 96-well microtiter plates and a standard 2fold microdilution method in MHIIB. Approximately $2 \times 10^{5}$ cells were inoculated and results were read after incubation at $37^{\circ} \mathrm{C}$ for $18 \mathrm{~h}$. For time killing assays, cells (10 $\mathrm{ml}$ ) were prepared as described above and exposed to FEP or CAZ added at a final concentration of $2 \times$ MIC of the K12 wild-type strain (i.e. FEP, $0.125 \mu \mathrm{g} / \mathrm{ml}$; CAZ, $0.25 \mu \mathrm{g} / \mathrm{ml})$. Series of 10 -fold dilutions were prepared in non-supplemented MHIIB every 30 min for 180 min and spread on $\mathrm{MH}$ agar plates containing ampicillin. Plates were incubated at $37^{\circ} \mathrm{C}$ for $18 \mathrm{~h}$ and colonies were manually counted. Colony forming units (CFU/ml) were calculated for each time point and plotted as the \% decrease in $\mathrm{CFU} / \mathrm{ml}$ in the presence as compared to in the absence of antibiotic.

omp35 and omp36 were PCR-amplified from a colony of E. aerogenes ATCC15038 by using specific primer pairs:

35-Fd 5'-GGAGGAATTCACCATGGTGAAGCGCAATATTCTGGCAGT-3' and 35-Rv 5'-CAGGTCGACTCTAGATTAGAACTGGTAAACGATACCAACC-3', and 36-Fd 5'-GGAGGAATTCACCATGGAAGTTAAAGTACTGTCCCTCCTG-3' and 36-Rv 5'-CAGGTCGACTCTAGATTAGAACTGGTAAACCAGGCC-3', respectively. PCR products were cloned under the $\mathrm{p}_{\mathrm{BAD}}$ promoter into the pBAD24 vector between the NcoI and XbaI restriction sites by using the InFusion cloning kit (Takara Bio USA Inc) according to the manufacturer instruction.

\section{Acknowledgments}

The research leading to these results was conducted as part of the Translocation consortium (www.translocation.com) and has received support from the Innovative Medicines Initiative Joint Undertaking under Grant Agreement no. 115525, resources which are composed of financial contribution from European Union's seventh framework programme (FP7/2007-2013) and EFPIA companies. S.A.G., M.P., L.F., 
J.W. are funded by EU FP7-PEOPLE-2013-ITN, Marie-Skłodowska Curie Translocation network Nr. 607694.

\section{Author Contribution}

MC, MW, MPGP, JHN, BvdB, J-MP, RS designed the research. SAG, MC, MW, MPGP, JHN, BvdB, RS wrote the manuscript. SAG, IB, MC performed the analysis of experimental data, the simulations, and designed the scoring function. LF, MP, MZ, JHN, BvdB performed the structure determination. LF, MP measured the LSA experiments. JW, MW performed the electrophysiology experiments. MM and J-MP performed whole-cell-based assays.

\section{Author information}

The atomic coordinates and structure factors have been deposited in the Protein Data Bank under accession numbers: 5O77, 5O79, 6ENE, 5O78, 5O9C.

\section{Supporting information}

Supplementary Figures 1-8 include orthologues structural alignment, porins architecture, porins size and electrostatic characterization, scoring function evaluation, electrophysiology data and permeability prediction versus already published data. Supplementary tables are also provided with additional information on crystallized porins, molecular properties of antibiotics, electrophysiology kinetic data on transport of antibiotics through porins.

\section{References}

1. Organization, W. H. Antimicrobial resistance: global report on surveillance. (2014).

2. O'Neill, J. (2016) Tackling drug-resistant infections globally: final report and recommendations. Rev. Antimicrob. Resist. 84 DOI 10.1016/j.jpha.2015.11.005

3. Blaskovich, M. A. T., Butler, M. S. \& Cooper, M. A. (2017) Polishing the tarnished silver bullet: the quest for new antibiotics. Essays Biochem. 61, 103114 DOI 10.1042/EBC20160077

4. Nikaido, H. (2003) Molecular basis of bacterial outer membrane permeability revisited. Microbiol. Mol. Biol. Rev. 67, 593-656 DOI 10.1128/MMBR.67.4.593-656.2003

5. Pagès, J. M., James, C. E. \& Winterhalter, M. (2008) The porin and the permeating antibiotic: a selective diffusion barrier in Gram-negative bacteria. Nat. Rev. Microbiol. 6, 893-903 DOI 10.1038/nrmicro1994

6. Masi, M., Réfregiers, M., Pos, K. M \& Pagès, J. M. (2017) Mechanisms of envelope permeability and antibiotic influx and efflux in Gram-negative bacteria. Nat. Microbiol. 2, 17001 DOI 10.1038/nmicrobiol.2017.1

7. $\quad$ Payne, D. J., Gwynn, M. N., Holmes, D. J. \& Pompliano, D. L. (2007) Drugs for bad bugs: confornting the challenges of antibacterial discovery. Nat. Rev. Drug Discov. 6, 29-40 DOI 10.1038/nrd2201

8. Tommasi, R., Brown, D. G., Walkup, G. K., Manchester, J. I. \& Miller, A. A. (2015) ESKAPEing the labyrinth of antibacterial discovery. Nat. Rev. Drug Discov. 14, 529-542 DOI 10.1038/nrd4572

9. Brown, E. D. \& Wright, G. D. (2016) Antibacterial drug discovery in the resistance era. Nature 529, 336-343 DOI 10.1038/nature17042.

10. Richter, M. F., Drown, B.S., Riley, A. P., Garcia, A., Shirai T., Svec R. L., \& Hergenrother, P. J. (2017) Predictive compound accumulation rules yield a 
broad-spectrum antibiotic. Nature 545, 299-304. DOI 10.1038/nature22308

11. O'Shea, R. \& Moser, H. E. (2008) Physiochemical Properties of Antibacterial Compounds: Implications for Drug Discovery. J. Med. Chem. 51, 2871-2878 DOI 10.1021/jm700967e

12. Brown, D. G., May-Dracka, T. L., Gagnon, M. M. \& Tommasi, R. (2014) Trends and exceptions of physical properties on antibacterial activity for grampositive and gram-negative pathogens. J. Med. Chem. 57, 10144-10161 DOI 10.1021/jm501552x

13. Nikaido, H., Rosenberg, E. Y. \& Foulds, J. (1983) Porin channels in Escherichia coli: studies with beta-lactams in intact cells. J. Bacteriol. 153, 232-240.

14. Kojima, S. \& Nikaido, H. (2013) Permeation rates of penicillins indicate that Escherichia coli porins function principally as nonspecific channels. Proc. Natl. Acad. Sci. U. S. A. 110, E2629-34 DOI 10.1073/pnas.1310333110

15. Kojima, S. \& Nikaido, H. (2014) High Salt Concentrations Increase Permeability Through OmpC Channels of Escherichia coli. J. Biol. Chem. 289, 26464-26473 DOI 10.1074/jbc.M114.585869

16. Nikaido, H. \& Rosenberg, E. Y. (1983) Porin channels in Escherichia coli: studies with liposomes reconstituted from purified proteins. J. Bacteriol. 153, 241-252.

17. Nestorovich, E. M., Danelon, C., Winterhalter, M. \& Bezrukov, S. M. (2002) Designed to penetrate: time-resolved interaction of single antibiotic molecules with bacterial pores. Proc. Natl. Acad. Sci. 99, 9789-9794. DOI 10.1073/pnas.152206799

18. Winterhalter, M. \& Ceccarelli, M. (2015) Physical methods to quantify small antibiotic molecules uptake into Gram-negative bacteria. Eur. J. Pharm. Biopharm. 95, 63-67 DOI 10.1016/j.ejpb.2015.05.006

19. Bajaj, H., Scorciapino, M. A., Moynié, L., Page, M. G. P., Naismith, J., Ceccarelli, M. \& Winterhalter, M. (2015) Molecular Basis of Filtering Carbapenems by Porins from $\beta$-Lactam-resistant Clinical Strains of Escherichia coli. J. Biol. Chem. 291, 2837-2847 DOI 10.1074/jbc.M115.690156

20. Westfall, D. A., Krishnamoorthy, G., Wollosecheck, D., Sarkar, R. \& Zgurskaya H.I. (2017) Bifurcation kinetics of drug uptake by Gram-negative bacteria. PLoS One. 12 (9): e0184671 DOI 10.1371/journal.pone.0184671

21. Nichols, W. W. (2017) Modeling the kinetics of the permeation of antibacterial agents into growing bacteria and its interplay with efflux. Antimicrob. Agents Chemother. 61 (10) e02576-16 DOI 10.1128/AAC.02576-16

22. Nagano, K. \& Nikaido, H. (2009) Kinetic behavior of the major multidrug efflux pump AcrB of Escherichia coli. Proc. Natl. Acad. Sci. U. S. A. 106 (14) 5854-5858 DOI 10.1073/pnas.0901695106

23. Lim, S. P. \& Nikaido, H. (2010) Kinetic parameters of efflux of penicillins by the multidrug efflux transporter AcrAB-TolC of Escherichia coli. Antimicrob. Agents Chemother. 54 (5) 1800-1806 DOI 10.1128/AAC.01714-09

24. Krishnamoorthy, G., Inga, V. L., Weeks, J. W., Wolloscheck D., Rybenkov, V. V. \& Zgurskaya H. I. (2017) Synergy between active efflux and outer membrane diffusion defines rules of antibiotic permeation into gram-negative bacteria. MBio. 8 (5) e01172-17 DOI 10.1128/mBio.01172-17

25. Cowan, S. W., Garavito, R. M., Jansonius, J. N., Jenkins, J. A., Karlsson, R., König, N., Pai, E. F., Pauptit, R. A., Rizkallah, P. J., Rosenbusch, J. P., Rummel, G. \& Schirmer, T. (1995) The structure of OmpF porin in a tetragonal 
crystal form. Structure. 3(10), 1041-50.

26. Baslé, A., Rummel, G., Storici, P., Rosenbusch, J. P. \& Schirmer, T. (2006)Crystal structure of osmoporin OmpC from E. coli at 2.0 Å. J. Mol. Biol. 362, 933-942 DOI 10.1016/j.jmb.2006.08.002

27. Acosta-Gutierrez, S., Scorciapino, M. A., Bodrenko, I. \& Ceccarelli, M. (2015) Filtering with Electric Field: The Case of E. coli Porins. J. Phys. Chem. Lett. 6, 1807-1812 DOI 10.1021/acs.jpclett.5b00612

28. Acosta-Gutiérrez, S., Bodrenko, I., Scorciapino, M. A. \& Ceccarelli, M. (2016) Macroscopic electric field inside water-filled biological nanopores. Phys. Chem. Chem. Phys. 18(13):8855-64. DOI 10.1039/C5CP07902K

29. Bajaj, H., Acosta-Gutiérrez, S., Bodrenko, I., Malloci, G., Scorciapino, M. A., Winterhalter, M. \& Ceccarelli, M (2017) Bacterial Outer Membrane Porins as Electrostatic Nanosieves : Exploring Transport Rules of Small Polar Molecules. ACS Nano, 11 (6), 5465-5473 DOI 10.1021/acsnano.6b08613

30. Bredin, J., Saint, N., Malléa, M., Dé, E., Molle, G., Pagès, J. M. \& Simonet, V. (2002) Alteration of pore properties of Escherichia coli OmpF induced by mutation of key residues in anti-loop 3 region. Biochem. J. 363, 521-528

31. Davin-Regli, A. \& Pagès, J. M. (2015) Enterobacter aerogenes and Enterobacter cloacae; Versatile bacterial pathogens confronting antibiotic treatment. Frontiers in Microbiology 6, 392 DOI 10.3389/fmicb.2015.00392

32. Mezzatesta, M. L., Gona, F. \& Stefani, S. (2012) Enterobacter cloacae complex: clinical impact and emerging antibiotic resistance. Future Microbiol. 7, 887-902 DOI 10.2217/fmb.12.61

33. Paczosa, M. K. \& Mecsas, J. (2016) Klebsiella pneumoniae: Going on the Offense with a Strong Defense. Microbiol. Mol. Biol. Rev. 80, 629-61 DOI 10.1128/MMBR.00078-15

34. Arunmanee, W., Pathania, M., Solovyova, A. S., Le Brun, A. P., Ridley, H., Baslé, A. \& Van den Berg, B. (2016) Gram-negative trimeric porins have specific LPS binding sites that are essential for porin biogenesis. Proc. Natl. Acad. Sci. 113, E5034-E5043 DOI 10.1073/pnas.1602382113

35. Pettersen, E. F., Goddard T. D., Huang, C. C., Couch, G. S., Greenblatt, D. M., Meng, E. C. \& Ferrin, T. E. (2004) UCSF Chimera-A Visualization System for Exploratory Research and Analysis. J Comput Chem 25, 1605-1612 DOI 10.1002/JCC. 20084

36. Gardiner, C. (2004) Handbook of stochastic methods: for physics, chemistry \& the natural sciences. Ser. Synerg. Vol. 13.

37. Scorciapino, M. A., Acosta-Gutiérrez, S., Benkerrou, D., D'Agostino, T., Malloci, G., Samanta, S., Bodrenko, I. \& Ceccarelli, M. (2017) Rationalizing the permeation of polar antibiotics into Gram-negative bacteria. J. Phys. Condens. Matter 29, 113001 DOI 10.1088/1361-648X/aa543b

38. Ghai, I., Pira, A., Scorciapino, M. A., Bodrenko, I., Benier, L., Ceccarelli, M., Winterhalter, M. \& Wagner, R. (2017) General Method to Determine the Flux of Charged Molecules through Nanopores Applied to $\beta$-Lactamase Inhibitors and OmpF. J. Phys. Chem. Lett. 8, 1295-1301 DOI 10.1021/acs.jpclett.7b00062.

39. Bangham, A. D., Hill, M. W. \& Miller, N. G. A. in Methods in Membrane Biology (ed. Korn, E. D.) 1-68 (Springer US, 1974). DOI 10.1007/978-1-46157422-4_1

40. Page, M. in Antibiotic Discovery and Development (ed. Dougherty, TJ and Pucci, M.) 79-117 (Springer, 2011). DOI 10.1007/978-1-464-1400-1_3 
41. Chapman, J. S. \& Georgopapadakou, N. H. (1988) Routes of Quinolone Permeation in Escherichia coli N10X. 32, 438-442.

42. Krishnamoorthy, G., Wolloscheck, D., Weeks, J. W., Croft, C., Rybenkov, V. V., Zgurskaya, H. I. (2016) Breaking the permeability barrier of Escherichia coli by controlled Hyperporination of the outer membrane. Antimicrob. Agents Chemother. 60, 7372-7381 DOI 10.1128/AAC.01882-16

43. Roche, O., Schneider, P., Zuegge, J., Guba, W., Kansy, M., Alanine, A., Bleicher, K., Danel, F., Gutknecht, E. M., Rogers-Evans, M., Neidhart, W., Stalder, H., Dillon, M., Sjögren, E., Fotouhi, N., Gillespie, P., Goodnow, R., Harris, W., Jones, P., Taniguchi, M., Tsujii, S., von der Saal, W., Zimmermann, G. \& Schneider, G. (2002) Development of a Virtual Screening Method for Identification of "Frequent Hitters" in Compound Libraries. J. Med. Chem. 45, 137-142 DOI 10.1021/jm010934d

44. Beaviss, A. D. \& Powers, M. F. (1989) On the Regulation of the Mitochondrial Inner Membrane Anion Channel by Magnesium and Protons *. J. Biol. Chem. 264, 17148-17155.

45. Dykens, J. A. \& Will, Y. (2008) Drug-Induced Mitochondrial Dysfunction. Drug-Induced Mitochondrial Dysfunction. DOI 10.1002/9780470372531

46. Guzman, L. M., Belin, D., Carson, M. J. \& Beckwith, J. (1995) Tight regulation, modulation, and high-level expression by vectors containing the arabinose P(BAD) promoter. J. Bacteriol. 177, 4121-4130.

47. Eren, E., Vijayaraghavan, J., Liu, J., Cheneke, B. R., Touw, D. S., Lepore, B. W., Indic, M., Movileanu, L. \& van den Berg, B. (2012) Substrate specificity within a family of outer membrane carboxylate channels. PLoS Biol 10, e1001242 DOI 0.1371/journal.pbio.1001242

48. Prilipov, A., Phale, P. S., Van Gelder, P., Rosenbusch, J. P. \& Koebnik, R. (1998) Coupling site-directed mutagenesis with high-level expression: Large scale production of mutant porins from E. coli. FEMS Microbiol. Lett. 163, 6572.

49. Kabsch, W. (2010) XDS. Acta Crystallogr D Biol Crystallogr 66, 125-132 DOI 10.1107/S0907444909047337

50. Murshudov, G. N., Vagin, A. A. \& Dodson, E. J. (1997) Refinement of macromolecular structures by the maximum-likelihood method. Acta Crystallographica Section D: Biological Crystallography 53, 240-255 DOI 10.1107/S0907444996012255

51. Emsley, P. \& Cowtan, K. (2004) Coot: Model-building tools for molecular graphics. Acta Crystallogr. Sect. D Biol. Crystallogr. 60, 2126-2132. DOI 10.1107/S0907444904019158

52. Battye, T. G. G., Kontogiannis, L., Johnson, O., Powell, H. R. \& Leslie, A. G. W. (2011) iMOSFLM: A new graphical interface for diffraction-image processing with MOSFLM. Acta Crystallogr. Sect. D Biol. Crystallogr. 67, 271-281 DOI 10.1107/S0907444910048675

53. Evans, P. (2006) Scaling and assessment of data quality. Acta Crystallogr D Biol Crystallogr 62, 72-82 DOI 10.1107/S0907444905036693

54. McCoy, A. J., Grosse-Kunstleve, R. W., Adams, P. D., Winn, M. D., Storoni, L. C. \& Read, R. J. (2007) Phaser crystallographic software. J. Appl. Crystallogr. 40, 658-674. DOI 10.1107/S0021889807021206

55. Davis, I. W., Murray, L. W., Richardson, J. S. \& Richardson, D. C. (2004) MolProbity: Structure validation and all-atom contact analysis for nucleic acids and their complexes. Nucleic Acids Res. 32, W615-W619 DOI 


\subsection{3/nar/gkh398}

56. Benz, R., Janko, K. \& Läuger, P. (1979) Ionic selectivity of pores formed by the matrix protein (porin) of Escherichia coli. Biochim. Biophys. Acta Biomembr. 551, 238-247 DOI 10.1016/0005-2736(89)90002-3

57. Baaken, G., Sondermann, M., Schlemmer, C., Rühe, J. \& Behrends, J. C. (2008) Planar microelectrode-cavity array for high-resolution and parallel electrical recording of membrane ionic currents. Lab Chip 8, 938 DOI 10.1039/ b800431e

58. Varma, S., Chiu, S. \& Jakobsson, E. (2006) The Influence of Amino Acid Protonation States on Molecular Dynamics Simulations of the Bacterial Porin OmpF. Biophys. J. 90, 112-123 DOI 10.1529/biophysj.105.059329

59. Phillips, J. C., Braun, R., Wang, W., Gumbart, J., Tajkhorshid, E., Villa, E., Chipot, C., Skeel, R. D., Kalé, L. \& Shulten K. (2005) Scalable molecular dynamics with NAMD. J. Comput. Chem. 26, 1781-1802. DOI 10.1002/jcc.20289

60. Harvey, M. J., Giupponi, G. \& Fabritiis, G. De. (2009) ACEMD: accelerating biomolecular dynamics in the microsecond time scale. J. Chem. Theory Comput. 5, 1632-1639 DOI 10.1021/ct9000685

61. Malloci, G., Vargiu, A.V., Serra, G., Bosin, A., Ruggerone, P. \& Ceccarelli, M. (2015) A Database of Force-Field Parameters, Dynamics, and Properties of Antimicrobial Compounds. Molecules 20, 13997-14021 DOI 10.3390/molecules200813997

62. Marvin 14.8.25.0. ChemAxon 2014. http://www. chemaxon. com.

63. Bayly, C. I., Cieplak, P., Cornell, W. \& Kollman, P. A. (1993) A well-behaved electrostatic potential based method using charge restraints for deriving atomic charges: the RESP model. J. Phys. Chem. 97, 10269-10280 DOI 10.1021/j100142a004

64. Wang, J., Wolf, R. M., Caldwell, J. W., Kollman, P. A. \& Case, D. A. (2004) Development and testing of a general amber force field. J. Comput. Chem. 25, 1157-1174 DOI 10.1002/jcc.20035

65. Humphrey, W., Dalke, A. \& Schulten, K. (1996) $\{$ VMD $\}--\{V\}$ isual $\{\mathrm{M}\}$ olecular $\{\mathrm{D}\}$ ynamics. J. Mol. Graph. 14, 33-38 DOI 10.1016/02637855(96)00018-5

66. James, C. E., Mahendram, K. R., Molitor, A., Bolla, J. M., Bessonov, A. N., Winterhalter, M. \& Pagès, J. M. (2009) How $\beta$-lactam antibiotics enter bacteria: A dialogue with the porins. PLoS One 4 DOI 10.1371/journal.pone.0005453 
TOC

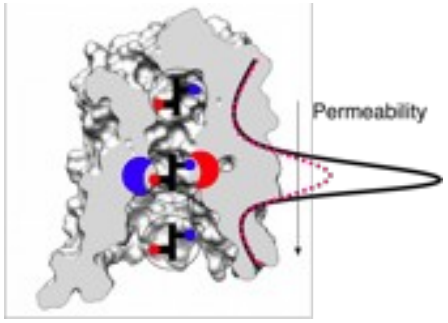

